\title{
The Research of Power System Short-term Load Forecasting
}

Jie Yi

\author{
North China Electrical Power University
}

\begin{abstract}
:
The precision of Power system short-term load forecasting directly is essential in running an efficient and economic power network.For the purpose of seeking reasonable predicting methods to predict electric load in a given period based on existing electricity capacity data,we select similar days of a given period and construct grey prediction model and BP neural network methods.
\end{abstract}

Key words:Short-term load forecasting; Grey prediction; BP neural network

\section{Introduction}

Power system short-term load forecasting is the most fundamental ground of optimization scheduling in power system,and an important component of daily operation management of electric power enterprises. The veracity of predicting results is crucial for power dispatching institution to develop generation schedule more reasonably and maintain the electric power supply and demand balance.As for State Grid,according to short-term load forecasting,reasonable scheduling of generating capacity,predicting requirements of power output for power plants and calculate changes in output of generator set can be achieved,therefore unit start up and shutdown can be arranged more economically and reasonably.In this way,not only can the power generation cost be reduced but also power system's security and stability can be raised.Short-term load forecasting,on the one hand,is a bias for power generation companies to make electric generation plans and quotations,on the other hand,it provides a basis for power supply company to make plans of power purchase and distribution.In addition,the set of the price of electricity demands accurate results of short-term load forecasting,ensuring great price competitiveness and profits.The power dispatch center is responsible for projected assessment of system adequacy.And the main work is to analyze and predict short-term supply and demand balance in power system,at the same time,the security of power system can be determined,thus,market members can acquire correct information,then run the electricity market smoothly. ${ }^{[1]}$ In conclusion,improving the precision is the focus and emphasis of current power system short-term load forecasting,accurate prediction of short-term load forecasting has become an important part of modern management of electrical system.

\section{Power system short-term load forecasting model principle}

To construct a reasonable model generating load prediction results in given period,we collect power load data in two areas within 6 years.Considering the local power load changes with time periodically,we anticipate similar days in given period and establish two short-term load forecasting models based on grey prediction and BP neural network respectively.Since then,we test the model precision to verify the model's rationality and reliability. 


\section{Grey prediction methods}

Grey prediction method predicts future trends through corresponding differential equation models,the model is based on data sequence with strong regularity deriving from original data.Data used in grey prediction is the result of backward method conducting on predicted values of the existing model.Grey prediction is based on grey model,we select the first order linear differential equation model to make predictions. $^{[2]}$

First, we accumulate input data to weaken the volatility and randomness of original random sequence,then a new data sequence is given.

$$
\left\{\begin{array}{l}
x_{1}(t)=\sum_{k=1}^{t} x_{0}(k), t=1,2, \cdots n \\
x_{1}(t+1)=\sum_{k=1}^{t+1} x_{0}(k) t=1,2, \cdots n
\end{array}\right.
$$

Then a first order linear differential equation can be obtained

$$
\frac{d x_{1}}{d t}+a x_{1}=u
$$

Where

$\mathrm{a}$ and $\mathrm{u}$ are under-determined coefficients and represent development coefficient and grey action.

The effective interval of $\mathrm{a}$ is $(-2,2)$, the matrix of a and $\mathrm{u}$ is $\hat{a}=\left(\begin{array}{l}a \\ u\end{array}\right)$. $x_{1}(t)$ can be determined on condition that a,u have been calculated.if $x_{1}(t)$ is known,the predicted value of ${ }^{x_{0}}$ can be given.Take the average of accumulated data,we can get the vector of $B$ and constant vector $Y_{n}$

$$
\begin{gathered}
B=\left[\begin{array}{c}
0.5\left(x_{1}(1)+x_{1}(2)\right) \\
0.5\left(x_{1}(2)+x_{1}(3)\right) \\
0.5\left(x_{1}(n-1)+x_{1}(n)\right)
\end{array}\right] \\
Y_{n}=\left(x_{0}(2), x_{0}(3), \cdots, x_{0}(n)\right)^{T}
\end{gathered}
$$

The grey parameter a can be obtained by least square estimation:

$$
\widehat{a}=\left(\begin{array}{l}
a \\
u
\end{array}\right)=\left(B^{T} B\right)^{-1} B^{T} Y_{n}
$$

Substitute parameter a into relation (4), $x_{1}$ can be calculated as follows:

$$
\widehat{x}_{1}(t+1)=\left(x_{0}(1)-\frac{u}{a}\right) e^{-a t}+\frac{u}{a}
$$


Since least square estimation provides approximate values,a is an approximation.On this basis,above equations are discretized,after subtraction,original sequence can be restored,the approximate data sequence is given as:

$$
\widehat{x}_{0}(t+1)=\widehat{x}_{1}(t+1)-\widehat{x}_{1}(t)
$$

Finally,precision of the grey prediction model is tested.Error index under consideration include residual $e_{0}(t)$, relative error $q(x)$ and little probability of error,which are respectively defined as

$$
\begin{gathered}
e_{0}(t)=x_{0}-\widehat{x}_{0}(t) \\
q(x)=\frac{e_{0}(t)}{x_{0}(t)} \\
P=P\left\{|e(t)| \prec 0.6745 s_{1}\right\}
\end{gathered}
$$

According to regression analysis and data analysis,we select data at different times in similar days as sample for grey prediction to predict electric power load.Data in similar days have approximate distribution rules with load data needs to be predicted,making the predicted results more reasonable. The selection of grey prediction model can be explained from two aspects.On the one hand,the sample data has strong regularity,the electric load also increases with exponential growth of economy.On the other hand,after researching into relative literature regarding various prediction methods,we find that grey prediction fits the prediction of load index following nonlinear trend well and is especially suitable when the sample data is few. As a result,we choose grey prediction as one prediction method.

To verify that grey prediction method is as precise as theory analysis,we testify the results of prediction model,then obtain grey prediction model's precision by comparing with the actual data.

\section{BP neural network}

The multi-layer perception model of BP neural network is constructed through imitating reactions of human neurons to external stimulus.Based on positive signal propagation and error back propagation,the intelligent network model processing nonlinear input is successfully established , and is modified through iterating ${ }^{[3]}$.

When design the BP neural network model,the number of hidden layer,hidden layer nodes,input layer node number,input layer node number and appropriate parameters need to be selected,transfer function,training methods and training parameters need to be determined.The BP neural network is used to train time series and electricity capacity,and then the function of power load can be obtained.

Robert Hecht-Nielsom had proved that BP network with only one hidden layer can be used as approximations to any continuous function in the closed interval.In other words, a three-layer BP neural network is enough to map data from $\mathrm{N}$-dimensional to M-dimensional.Therefore,BP network with one hidden layer is commonly used.The number of hidden layer nodes is a key problem in design.It depends on the nonlinear 
degree between input and output,in other words, the hidden unit numbers have a direct relationship with the number of input and output layer.Generally,if the hidden unit is too few,the network won't be trained well,it cannot identify a new sample; while if there are too many hidden layer nodes,the network is able to classify training data set,fault tolerance of the system is reduced.But on the other hand,even input mode change slightly,the network will fail to identify it,and the training time will has a significant increase.The problem of over-fitting tends to appear.Therefore,it's a complex problem to choose the hidden layer nodes.Following formulas can be referred to ${ }^{[4]}$ :

$$
K \prec \sum_{i=1}^{N} C\left(\begin{array}{c}
h \\
i
\end{array}\right)
$$

Where

$\mathrm{K}$ is sample size, $h$ represents the hidden unit numbers, $\mathrm{N}$ is the number of input units

$$
h=\sqrt{(N+M)}+\alpha
$$

Where

$M$ is the number of output units, $\mathrm{N}$ is the number of input units, $\alpha$ is the constant between 1 and 10 .

$$
h=\log _{2} N
$$

Where

$\mathrm{N}$ is the number of input units.

The inputs to the neural networks are different times,tansig, purelin are determined as the transfer function,the outputs of the network are predicted electric loads. When training results become steady,the electric load growth function can then be obtained.According to existing relevant data in similar days,electric load can be predicted accurately.Error analysis is given to show an error within 10MW.

\section{Conclusion}

Comparing test results for accuracy,we can see that relative errors of grey prediction and the BP neural network are both less than 0.06.The BP neural network has a slight edge in accuracy.The concepts of similar days and diversity are used to select data source. Finally,relatively accurate predicting results of electric load can be obtained.

\section{References}

[1]Xu Hongrui. The short-term load forecasting of power system considering the meteorological factors[D].North China Electric Power University (Hebei),2009.

[2]Zhuo Jinwu. Application of MATLAB in mathematical modeling [M]. Beihang University press, 2011.

[3]Chen Ming. MATLAB neural network principle and examples of [M]. Tsinghua University press, 2013

[4]Li Lixia, Tong Wang,. Design of the BP neural network [J]. Modern preventive medicine,2005,(02):128-130. 